\title{
HIP 60350: A supernova ejected hyper-runaway star?
}

Andreas Irrgang*1, Norbert Przybilla ${ }^{1}$, Ulrich Heber ${ }^{1}$, M. Fernanda Nieva ${ }^{2}$, Sonja Schuh $^{3}$

${ }^{1}$ Dr. Karl Remeis-Sternwarte Bamberg \& ECAP, Bamberg

${ }^{2}$ Max Planck Institute for Astrophysics, Garching

${ }^{3}$ Georg-August-Universität Göttingen, Göttingen

E-mail: andreas.irrgangesternwarte.uni-erlangen.de

Halo runaway stars, i.e., young, massive, high-velocity stars in the halo of our Galaxy, are widely supposed to be the result of an ejection event from the Galactic disk. The three most likely ejection scenarios are tidal disruptions of binary systems by the supermassive black hole at the Galactic center, dynamical interactions in star clusters, e.g., binary-binary encounters, or a supernova explosion disrupting a binary system. Here, we present a detailed spectroscopic and kinematic analysis of the runaway B star HIP 60350 to discuss the three competing mechanisms. Using spectrum synthesis techniques that allow for deviations from local thermal equilibrium, we derived chemical elemental abundances from high resolution spectra. The analysis of HIP 60350 revealed an outstanding high Galactic rest-frame velocity of $530 \pm 35 \mathrm{~km} \mathrm{~s}^{-1}$ slightly exceeding the local Galactic escape velocity and thus qualifying the star as a hyper-runaway candidate, i.e., an unbound runaway star. The kinematically predicted birthplace within the disk is located $\sim 6 \mathrm{kpc}$ away from the Galactic center excluding this region as the star's spatial origin and in this way the involvement of the central supermassive black hole. Instead, the obtained chemical abundance pattern shows hints of $\alpha$-element enhancement being characteristic of supernova debris. HIP 60350 might be the surviving secondary of a massive, close binary system disrupted by the primary's supernova explosion.

11th Symposium on Nuclei in the Cosmos

19-23 July 2010

Heidelberg, Germany.

\footnotetext{
* Speaker.
} 


\section{Hyper-runaway stars: Linking runaway stars to hyper-velocity stars}

Young, massive stars are usually found close to the Galactic plane, preferentially in open clusters and associations. Some of them, however, are observed at high Galactic latitudes far away from any star-forming regions. Since no gas clouds are known in the halo that have a sufficient density to form massive stars, these stars are supposed to have formed in the Galactic disk, and afterwards migrated outwards as so-called runaway stars. They are thought to have been ejected from their place of birth and accelerated to high velocity either by dynamical processes in star clusters, e.g. during the initial dynamical relaxation ([10]) or in many-body encounters ([7], see animated gif "NIC XI_151_a1.gif "), or by means of a supernova explosion disrupting a binary system ([2], see animated gif "NIC XI_151_a2.gif"). Their ejection velocities are typically well below the local Galactic escape velocity contrary to so-called hyper-velocity stars, which are unbound to the Galaxy and supposed to be produced by interaction with the supermassive black hole at the Galactic center ("Hills mechanism", [5], see animated gif "NIC XI_151_a3.gif"). However, Heber et al. ([4]) and Przybilla et al. ([11]) have shown that the unbound B-type star HD 271791 cannot originate from the Milky Way's center, excluding the supermassive black hole scenario as ejection event. Instead, due to its current 3D kinematics it could be shown that the birthplace of HD 271791 lies at the metal-poor rim of the Galactic disk, a conclusion in total agreement to the subsolar iron abundance revealed by a detailed spectroscopic analysis ([11]). Therefore HD 271791 is the first discovered hyper-runaway star, i.e. a hyper-velocity star not stemming from the Galactic center. The striking enhancement of $\alpha$-elements in the atmosphere of HD 271791 can be explained plausibly by the capture of nucleosynthesis products from a supernova, implying that the star might be the surviving secondary of a close, massive binary system in which the primary underwent a supernova explosion. HD 271791 could then either be ejected during the course of the explosion ([11]) or via strong dynamical three- or four-body encounters involving the post-supernova binary ([3]).

\section{Scientific rationale and goals}

Investigating runaway stars allows conclusions on the dynamics during many-body encounters or supernova explosions to be drawn, constraints on the structure and gravitational potential of the Galaxy to be derived and the question whether in situ star formation in the halo is possible or not to be addressed. Moreover, the example of HD 271791 has shown that extreme runaway stars are suited to obtain insights into stellar nucleosynthesis during supernova explosions from an independent and so far not exploited indicator. Analysis of the kinematic and spectroscopic properties of runaway stars to distinguish between the different formation channels and precise abundance analyses of supernova-ejected runaway stars are therefore important scientific goals, the latter to derive observational constraints on theoretical supernova models.

\section{HIP 60350}

HIP 60350 is a mid-B-type star located $\sim 3 \mathrm{kpc}$ above the Galactic disk and shares many properties with HD 271791, in particular it has the second largest known Galactic rest-frame velocity among all runaway stars ([8]). Consequently, there is a high probability for an extreme ejection event in the past calling for a detailed analysis of HIP 60350 as well. 


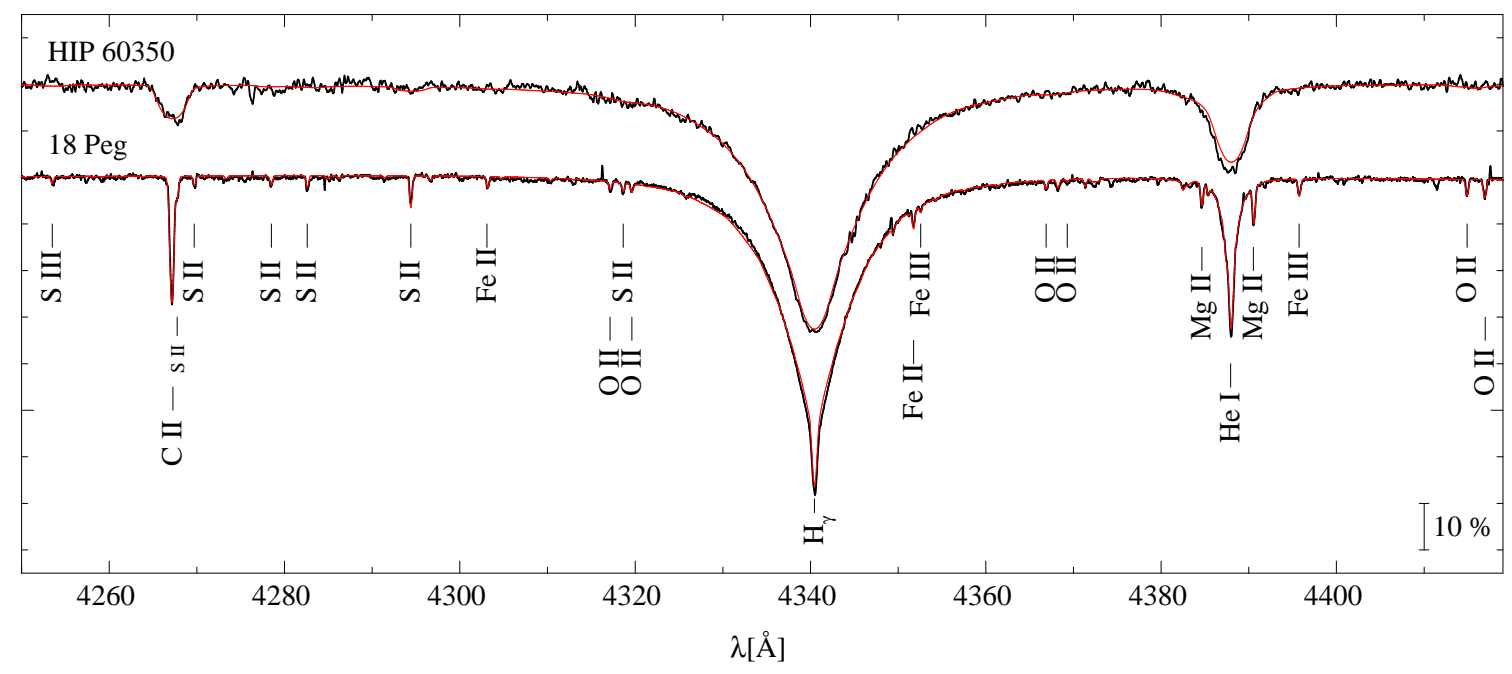

Figure 1: Comparison of the final spectrum synthesis for HIP 60350/18 Peg (red line) with observation (black line) for an exemplary region around $\mathrm{H}_{\gamma}$. Taken from Irrgang et al. [6].

\section{Analysis}

\subsection{Spectroscopy}

The spectroscopic investigation is based on a high-resolution spectrum obtained at the 9.2 meter Hobby-Eberly Telescope at McDonald Observatory with resolving power $\lambda / \Delta \lambda=15000$ and signal-to-noise ratio $\mathrm{S} / \mathrm{N} \sim 140$ in the blue visual range. The quantitative analysis - encompassing determination of atmospheric parameters like effective temperature $T_{\text {eff }}$, logarithmic surface gravity $\log g\left(g\right.$ in $\left.\mathrm{cm} \mathrm{s}^{-2}\right)$, projected rotational velocity $v \sin i$ as well as elemental abundances via detailed line-profile analysis and fitting of the spectral energy distribution - uses techniques that allow for deviations from local thermal equilibrium which introduce non-negligible effects in the spectra of B-type stars. In order to exclude systematic biases, a differential abundance analysis with respect to the normal B-type star 18 Peg was performed. The latter was chosen because of similar atmospheric parameters, low $v \sin i$ reducing the blending of lines and the high quality of the available spectrum. For both stars, the overall agreement between observation and theory is quite good (see Fig. 1). Stellar parameters (mass, age) were then constrained by comparing the location of HIP 60350 in a $\left(T_{\text {eff }}, \log g\right)$ diagram with evolution tracks calculated by Schaller et al. ([14]).

\subsection{Kinematics}

To study the kinematics of the star, knowledge of all three components of current position and velocity is necessary. From spectroscopic quantities, the distance $d$ to the star can be derived using the method described in Ramspeck et al. ([13]). The radial velocity $v_{\text {rad }}$ is achievable via the Doppler shift of spectral lines. Tangential angular motions are also available by means of Hipparcos space satellite measurements of the star's position over the years transforming into absolute velocities by multiplication with the distance. Hence the current 3D kinematics is completely known allowing to trace back HIP 60350's orbit to the Galactic plane (see animated gif "NIC XI_151_a4.gif") to check whether its kinematic properties are consistent with a Galactic disk runaway nature at all. In order to do so, the Galactic gravitational potential of Allen \& Santillan ([1]) as well as the numerical code of Odenkirchen \& Brosche ([9]) is used. Uncertainties in the 

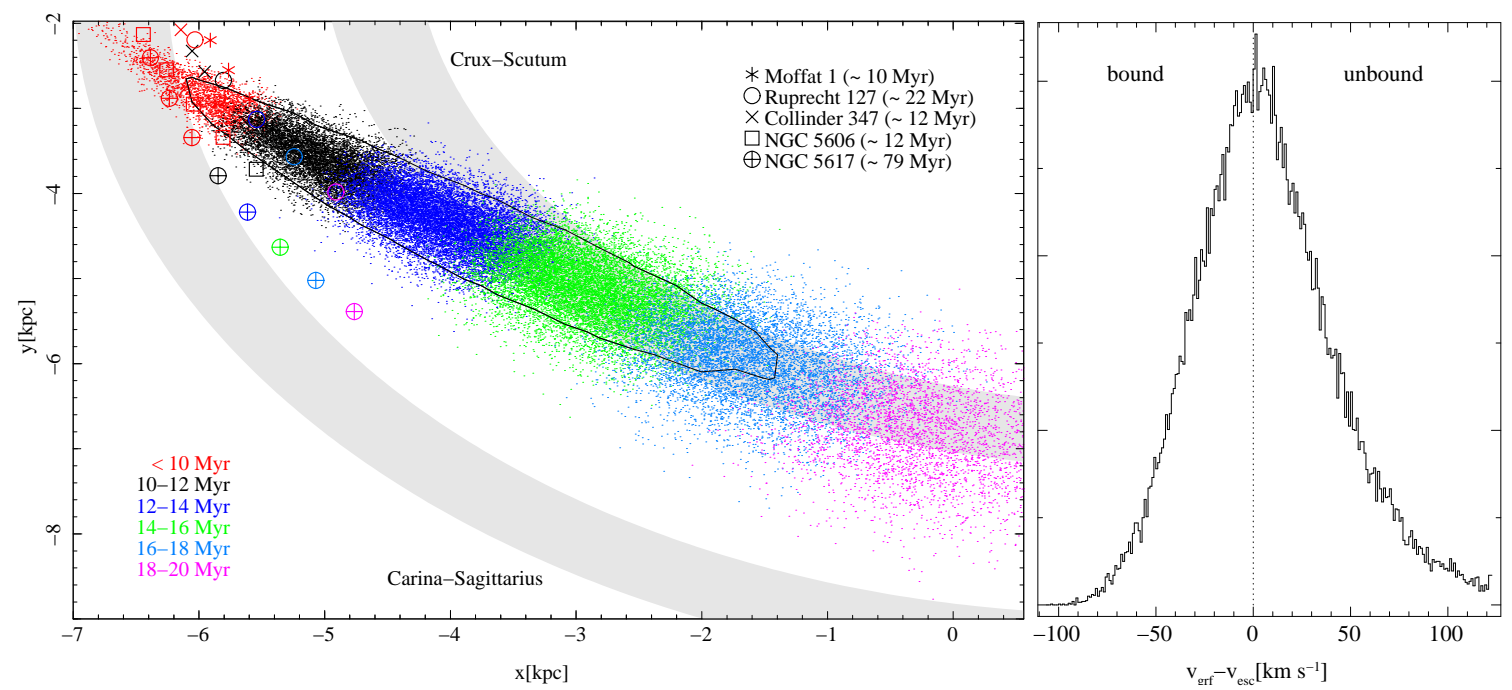

Figure 2: Left panel: Galactic disk intersection region of the Monte Carlo sample of orbits (dots). Additionally, the trajectories of possible host clusters are depicted. The travel time's color code is given in the lower left corner. A right-handed, non-rotating frame of reference with the Galactic center at the origin, Galactic north pole in positive $z$-direction and the Sun's current position $(-8.0,0,0) \mathrm{kpc}$ is used. The contour line marks the $1 \sigma$ region, i.e., it contains $\sim 68 \%$ of all dots. The gray shaded regions schematically represent the locus of two spiral arms at the time of disk intersection. Right panel: histogram showing the distribution of (un)bound trajectories in the sample. The abscissa is the difference between current space velocity $v_{\text {grf }}$ and the local escape velocity $v_{\text {esc }}$.

current position and velocity are accounted for by applying a Monte Carlo method which varies simultaneously and independently all parameters assuming a Gaussian distribution. In total, 50000 trajectories have been computed.

\section{Results}

The investigation of the star's motion leads to four important results ${ }^{1}$ : I) The travel time $T_{\text {flight }}=14 \pm 3 \mathrm{Myr}$ is smaller than the stellar evolutionary age $T_{\text {evol }}=45_{-30}^{+15} \mathrm{Myr}$ being a necessary condition for a runaway nature. II) A very high ejection velocity of $v_{\mathrm{ej}}=394 \pm 42 \mathrm{kms}^{-1}$ was derived. III) The Galactic center and hence the Hills mechanism can be ruled out as HIP 60350's origin (see left panel of Fig. 2). VI) The star's Galactic rest frame velocity may exceed its local Galactic escape velocity (see right panel of Fig. 2) qualifying HIP 60350 as hyper-runaway candidate.

For the first time, a quantitative spectroscopic analysis of HIP 60350 was based on non-local thermodynamic equilibrium techniques allowing to significantly revise atmospheric and stellar parameters. The results nicely reproduce the observed spectrum (see Fig. 1). In order to unveil the star's history, chemical elemental abundances were derived, too. With respect to the reference star 18 Peg, HIP 60350 shows hints for an enhancement of elements produced during core-collapse supernova explosion (see Fig. 3) leading to the conjecture that the star might be the surviving sec-

\footnotetext{
${ }^{1}$ The precision of the kinematic study will drastically improve as soon as astrometric data from ESA's cornerstone mission Gaia will become available.

${ }^{2}$ Corrected for Galactic rotation.
} 


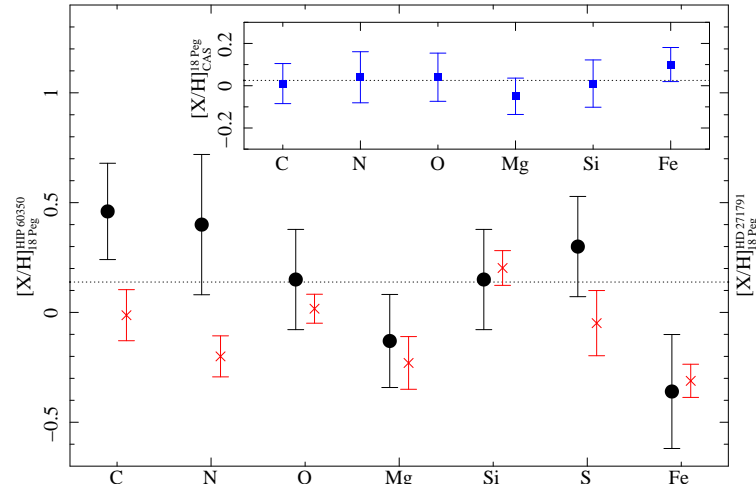

Figure 3: Metal abundances of HIP 60350 (filled black circles) and HD 271791 (red crosses, Przybilla et al. [11]) relative to the reference star 18 Peg. The notation $[\mathrm{X} / \mathrm{H}]_{\mathrm{B}}^{\mathrm{A}} \equiv \log (\mathrm{X} / \mathrm{H})_{\mathrm{A}}-$ $\log (\mathrm{X} / \mathrm{H})_{\mathrm{B}}$ is used. The inset shows the abundances of 18 Peg (filled blue squares) relative to the cosmic abundance standard by Przybilla et al. ([12]) revealing that the star is representative for the solar neighborhood. The dotted lines mark the average of the filled circles or squares, respectively. Taken from Irrgang et al. [6].

ondary of a massive, close binary system disrupted by the primary's supernova explosion. However, the results do not rule out a dynamical ejection event.

The examples of HD 271791 and HIP 60350 indicate that high precision, quantitative abundance analyses of supernova-ejected runaway stars allow to infer observational constraints on theoretical supernova models.

\section{References}

[1] Allen, C. \& Santillan, A., An improved model of the galactic mass distribution for orbit computations, Rev. Mex. AA 22 (1991) 255

[2] Blaauw, A., On the origin of the $O$ - and B-type stars with high velocities (the "run-away" stars), and some related problems, Bull. Astron. Inst. Neth. 15 (1961) 265

[3] Gvaramadze, V. V., HD 271791: dynamical versus binary-supernova ejection scenario, MNRAS 395 (2009) L85

[4] Heber, U. et al., The B-type giant HD 271791 in the Galactic halo. Linking run-away stars to hyper-velocity stars, A\&A $\mathbf{4 8 3}$ (2008) L21

[5] Hills, J. G., Hyper-velocity and tidal stars from binaries disrupted by a massive Galactic black hole, Nature 311 (1988) 687

[6] Irrgang, A. et al., The Nature of the Hyper-Runaway Candidate HIP 60350, ApJ 711 (2010) 138

[7] Leonard, P. J. T. \& Duncan, M.J., Runaway stars from young star clusters containing initial binaries. I - Equal-mass, equal-energy binaries, AJ 96 (1988) 222

[8] Maitzen, H. M. et al., HIP 60350: an extreme runaway star, A\&A 339 (1998) 782

[9] Odenkirchen, M. \& Brosche, P., Orbits of galactic globular clusters, Astronom. Nachr. 313 (1992) 69

[10] Poveda, A., Ruiz, J. \& Allen, C., Run-away Stars as the Result of the Gravitational Collapse of Proto-stellar Clusters, Bol. Obs. Tonantzintla Tacubaya 4 (1967) 86

[11] Przybilla, N. et al., HD 271791: An Extreme Supernova Runaway B Star Escaping from the Galaxy, ApJ 684 (2008) L103

[12] Przybilla, N., Nieva, M. F. \& Butler, K., A Cosmic Abundance Standard: Chemical Homogeneity of the Solar Neighborhood and the ISM Dust-Phase Composition, ApJ 688 (2008) L103

[13] Ramspeck, M. et al., Early type stars at high galactic latitudes. II. Four evolved B-type stars of unusual chemical composition, $A \& A 379$ (2001) 235

[14] Schaller, G. et al., New grids of stellar models from 0.8 to 120 solar masses at $Z=0.020$ and $Z=$ $0.001, A \& A S 96$ (1992) 269 